\title{
From a one-dimensional crystal to a one-dimensional liquid: A comprehensive dynamical study of $C_{60}$ peapods
}

\author{
Colin Bousige \\ Institut Laue Langevin, 6 rue Jules Horowitz, B.P. 156, F-38042 Grenoble Cedex 9, France and Laboratoire de Physique des Solides, \\ UMR CNRS 8502, Université Paris-Sud, F-91405 Orsay, France
}

Stéphane Rols, ${ }^{*}$ Jacques Ollivier, Helmut Schober, and Peter Fouquet Institut Laue Langevin, 6 rue Jules Horowitz, B.P. 156, F-38042 Grenoble Cedex 9, France

Giovanna G. Simeoni

Forschungsneutronenquelle Heinz Maier-Leibnitz, Technische Universität München, 85747 Garching, Germany

Viatcheslav Agafonov

GREMAN, UMR CNRS 6157, F-37200 Tours, France

Valery Davydov

Institute of High Pressure Physics of the RAS, R-142092 Troitsk, Moscow Region, Russian Federation

Yoshiko Niimi and Kazutomo Suenaga

Nanotube Research Center, National Institute of Advanced Industrial Science and Technology (AIST), Central 5, Tsukuba 305-8565, Japan

\author{
Hiromichi Kataura \\ Nanosystem Research Institute, National Institute of Advanced Industrial Science and Technology (AIST), Central 4, \\ Higashi 1-1-1, Tsukuba, Ibaraki 305-8562, Japan \\ Pascale Launois ${ }^{\dagger}$ \\ Laboratoire de Physique des Solides, UMR CNRS 8502, Université Paris-Sud, F-91405 Orsay, France \\ (Received 25 January 2013; revised manuscript received 6 April 2013; published 22 May 2013)
}

\begin{abstract}
We report an inelastic neutron-scattering investigation of the longitudinal acoustic modes of $\mathrm{C}_{60}$ chains confined inside single walled carbon nanotubes. We take advantage of the orientations of the chains within the plane of the pellet sample to isolate their scattering signatures in the $(Q, \omega)$ space, which we follow as a function of temperature from $260 \mathrm{~K}$ up to $1100 \mathrm{~K}$. The results show the progressive evolution of the confined chain from a one-dimensional (1D) crystal to a linear liquid, the transition occurring within a temperature range of $\sim 150 \mathrm{~K}$ centered around $600 \mathrm{~K}$. The comparison of the data obtained on monomer and polymer peapods allows extracting the speed of sound in the monomer crystalline chains $\left(v_{\text {mono }}=3.5 \mathrm{~km} \mathrm{~s}^{-1}, v_{\text {poly }} / v_{\text {mono }}=1.7\right)$. We find that the sound velocity is further reduced by half in the liquid state which reveals that the melting is not only due to harmonic additive thermal fluctuations, but that anharmonic terms in the intermolecular potential play an important role at high temperatures.
\end{abstract}

DOI: 10.1103/PhysRevB.87.195438 PACS number(s): 61.05.F-, 61.48.-c, 65.80.-g, 68.65.-k

\section{INTRODUCTION}

The first observation of $\mathrm{C}_{60}$ fullerene chains inside single walled carbon nanotubes (SWNTs) ${ }^{1}$-also known as "peapods"- has led to a vast amount of theoretical studies. Certainly motivated by the elegance of the perfect autoassembly of what was commonly referred to as one-dimensional (1D) (nanotube) and $0 \mathrm{D}\left(\mathrm{C}_{60}\right)$ forms of carbon, a large number of theoretical studies have been published, many of them concerned with the electronic properties of the peapods while some others were devoted to their thermodynamics. ${ }^{2-8}$ On the experimental side, the majority of the investigations published so far are concerned with electron microscopy and diffraction, as well as Raman spectroscopy, x-ray diffraction, or NMR. Most of them are related to the modifications of the optical properties $^{9}$ and to the evolution of the structure under different conditions, like pressure ${ }^{10-14}$ or doping. ${ }^{15}$ Few publications report on the fluctuations and on the low-frequency dynamics of confined $\mathrm{C}_{60}$ molecules. In particular, it was found that confining the $\mathrm{C}_{60}$ on a chain results in a large temperature downshift of the so-called "order-disorder" or orientational melting transition. The latter transition indeed occurs at $255 \mathrm{~K}$ in solid (fcc) $\mathrm{C}_{60},{ }^{16-18}$ while it was observed around $100 \mathrm{~K}$ in peapods. ${ }^{19,20}$ The low dimensionality of peapods was also recently reported by us to be at the origin of the progressive translational melting of the confined $1 \mathrm{D} \mathrm{C}_{60}$ chains at high temperature, ${ }^{21}$ a behavior almost perfectly described by the Takahasi-Gúrsey model introduced by Girifalco and Hodak. ${ }^{7}$ In particular, we have shown that the high filling ratio of our samples (97\% peapods internal volume occupied by $\mathrm{C}_{60}$ chains) prevents some predicted clusterization of the chains to occur when increasing the temperature. ${ }^{7,8}$ In this 
case, the melting process was established to result from the sole increase of thermal longitudinal fluctuations within the chain. What remains unclear at the moment is to what extent the anharmonicity of the inter- $\mathrm{C}_{60}$ potential plays a role in the transformation. A full understanding of the physics at the origin of the 1D translational melting phenomenon therefore requires the ability to grasp the full dynamics of the confined chain, i.e., rotations and translations.

In this paper, we extend our previous investigations of the $1 D$ translational melting by probing directly the translational dynamics of the confined $\mathrm{C}_{60}$ chain using inelastic neutron scattering (INS). In the whole temperature range investigated in this study $(T \in[260,1100] \mathrm{K})$, the peapods are in the "orientational disordered" phase, with the $\mathrm{C}_{60}$ molecules undergoing fast isotropic rotations. ${ }^{19,20,22}$ In this phase, there is no correlation between the molecular orientations along the chain. The melting transition that will be discussed in the following thus concerns the loss of long-distance correlation between the $\mathrm{C}_{60}$ center of mass along the chain with increasing temperature. Therefore, the 1D melting transition discussed in the rest of this paper is different in nature from the orientational melting transition occurring at $\sim 100 \mathrm{~K}$.

As will be described along the paper, we mainly focused on the investigation of the physics of "monomer" peapods, i.e., peapods for which the confined fullerenes are packed through van der Waals interactions inside the nanotubes. It is well known that under specific conditions of pressure and temperature, one can synthesize metastable "polymer" peapods, for which adjacent $\mathrm{C}_{60}$ are linked via $(2+2)$ covalent bonds. ${ }^{10} \mathrm{In}$ our experimental approach, we use the large difference in the magnitude of the force constants between the monomer and polymer bonds to derive quantitative information related to the monomer peapods. This is based on the reasonable assumption that the nanotube host and the low dimensionality of the chain do not have a strong influence on the covalent $\mathrm{C}_{60}$ bonds of the polymer peapods. We therefore assume that the linear stiffness of the confined polymer chain is the same as that measured in some well-known $\mathrm{C}_{60}$ crystalline polymer phases, and we consider that the sound velocity, for example, is equivalent in both systems to a very good approximation.

The study presented in this paper is articulated as follows: First, we describe the sample preparation and the experimental setup we used. Then, we discuss two analytical models (1D harmonic crystal and 1D harmonic liquid), which are the basis of the data analysis. Finally, we present the experimental measurements in the $[260,1100]-\mathrm{K}$ temperature range and discuss them. The comparison of data coming from the two systems (monomer and polymer peapods) allows extracting quantitative information such as the speed of sound in monomer chains or the mean system size. Our conclusions follow.

\section{EXPERIMENT}

\section{A. Sample preparation}

The peapod samples were elaborated with the aim of being used for INS measurements. Therefore, extreme care not to introduce any hydrogenated pollutant was taken during their preparation. Nanotubes of the SO type were purchased from the Meijo company, ${ }^{23}$ and the fullerenes are MER (Ref. 24) sublimed $\mathrm{C}_{60} 99.9 \%$ graded. Following the method developed in Ref. 25, carbon nanotubes under the form of buckypapers were first heated at $600{ }^{\circ} \mathrm{C}$ under dynamic vacuum in order to extract any solvent impurities. They were further heated in air at $500{ }^{\circ} \mathrm{C}$ in order to selectively open them around the reactive sites located at the tips and the defects of the tubes. The tubes and the fullerenes were then sealed in a Pyrex tube under a $10^{-6}$-mbar vacuum, and subsequently heated at $550{ }^{\circ} \mathrm{C}$ during 8 days. Buckypapers with fullerenes inside nanotubes were then washed in toluene and heated again at $600{ }^{\circ} \mathrm{C}$ under dynamic vacuum in order to remove the solvent and the fullerenes that did not enter the tubes. Prior to the neutron experiment, the $1.8-\mathrm{g} \mathrm{C}_{60}$ peapod buckypapers were pressed into pellets and heated at $100{ }^{\circ} \mathrm{C}$ while pumping in order to remove water molecules that could have adsorbed on the sample postsynthesis.

Part of the peapods synthesized as above were then polymerized. Polymerization of $\mathrm{C}_{60}$ molecules inside of SWNTs was achieved by high-pressure high-temperature treatment (HPHTT) of the monomer state of the peapods. Cold-pressed pellets of the pristine monomer $\mathrm{C}_{60}$ peapods $(12 \mathrm{~mm}$ diameter and $6 \mathrm{~mm}$ height) embedded in an insulating capsule of hexagonal boron nitride were inserted into a graphite heater and then placed inside the high-pressure cell. HPHTT of the samples was carried out in a high-pressure apparatus of "toroid" type. ${ }^{26}$ The experimental procedure involved loading of the apparatus to the pressure of $2.5 \mathrm{GPa}$, followed by the heating and isothermal holding of the samples at temperature of $280{ }^{\circ} \mathrm{C}$ during $30 \mathrm{~min}$. This process creates $(2+2)$ "cycloadditive" covalent bonds all along the $\mathrm{C}_{60}$ chains. This high-pressure state was preserved by quenching the sample to room temperature under pressure. At ambient condition, the polymer and monomer samples were characterized by Raman spectroscopy, scanning, and transmission electron microscopy (SEM and TEM) and X-ray diffraction (XRD) (see Fig. 1).

The structural analysis of the peapods give the following characteristics: ${ }^{21,28}$ nanotubes diameter Gaussian distribution centered at $14.2 \AA$ with $2 \AA$ full width at half maximum (FWHM); bundle mean size: $\sim 85$ tubes; inter- $\mathrm{C}_{60}$ distance: $9.9 \AA$ for monomer and $9.2 \AA$ for polymer; nanotubes filling ratio by $\mathrm{C}_{60}$ molecules (monomer): $97 \%$. The length of the nanotubes varies between 1 and $5 \mu \mathrm{m} .{ }^{23}$ The quantitative analysis of the $\mathrm{x}$-ray-diffraction profile of the monomer and polymer $\mathrm{C}_{60}$ peapods further shows that, for both samples, the peapods take all possible orientations within the plane of the pellet but have their long axis lying in this plane with $\mathrm{a} \pm 30^{\circ}$ off-plane distribution. ${ }^{12,28,29}$ We refer to this configuration as a "partial" 2D orientation of the peapods.

\section{B. Inelastic neutron scattering}

The inelastic neutron scattering (INS) investigations were performed on the cold neutron time of flight (TOF) spectrometers IN5 at the ILL (Grenoble, France) and TOFTOF at FRMII (Garching, Germany), allowing us to monitor the evolution of the density of states of the 1D acoustic phonons as a function of temperature. Both instruments are very similar in terms of signal-to-noise ratio. A cryofurnace was installed on IN5, allowing varying the temperature between 260 and $420 \mathrm{~K}$ (standard ILL orange cryofurnace), whereas a standard high-temperature vacuum furnace (20 mbar helium pressure 


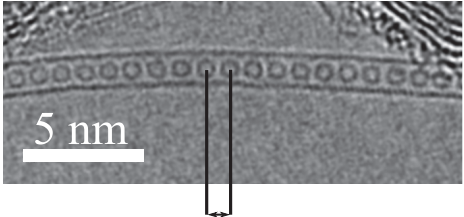

Monomer: $9.9 \AA$
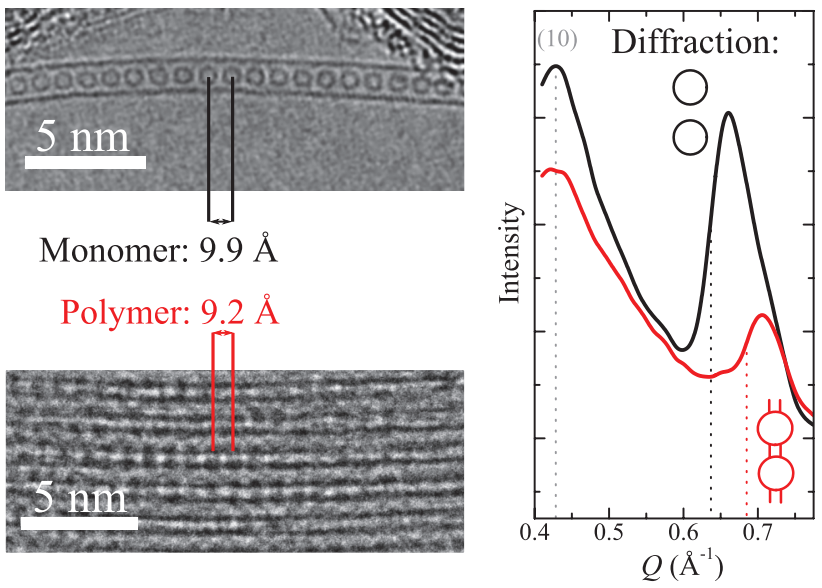

FIG. 1. (Color online) Left: TEM images of monomer (top) and polymer (bottom) peapods (monomer and polymer peapods are usually found in bundles, but a better viewing was offered by this isolated monomer peapod). Right: X-ray diffraction of monomer and polymer samples. The peak around $0.4 \AA^{-1}$ labeled (10) is the first nanotube bundle peak (peapods are organized in bundles on a two-dimensional hexagonal lattice which leads to Miller indices $(h k)$ in reciprocal space; here $h=1$ and $k=0$ ). The one around $0.65 \AA^{-1}$ arises from the $1 \mathrm{D} \mathrm{C}_{60}$ chains. ${ }^{27}$ The mean distance $d$ between the $\mathrm{C}_{60}$ molecules is given by the position $Q_{1}$ of the inflexion point of the sawtooth peak just after $0.6 \AA^{-1}\left(Q_{1}=2 \pi / d\right) .{ }^{21}$ The polymerization results in a clear shift of the $\mathrm{C}_{60}$ correlation peak towards higher $Q$ values: ${ }^{10-12} Q_{1}^{\text {mono }}=0.637 \AA^{-1}$ and $Q_{1}^{\text {poly }}=0.685 \AA^{-1}$.

corrected at each temperature) was installed on TOFTOF for measurements in the $[300,1043]-\mathrm{K}$ range. On both instruments, incident neutrons with wavelength of $8 \AA$ were used, giving an elastic resolution of $25 \mu \mathrm{eV}$ and a dynamical range limited to energy transfers $\hbar \omega \in[0,1] \mathrm{meV}$ on the Stokes side, and elastic scattering vectors $Q$ within the $[0.4,1.4] \AA^{-1}$ range. Here the scattering vector $\boldsymbol{Q}$ is defined as $\boldsymbol{Q}=\boldsymbol{k}-\boldsymbol{k}^{\prime}$ with $\boldsymbol{k}\left(\boldsymbol{k}^{\prime}\right)$ the incident (scattered) neutron wave vector; energy transfer is defined as $\hbar \omega=E-E^{\prime}$ with $E\left(E^{\prime}\right)$ the incident (scattered) neutron energy. Experimental resolutions were measured at $2 \mathrm{~K}$ on IN5 and $300 \mathrm{~K}$ on TOFTOF. After the measurements, the data were subsequently background corrected and normalized to vanadium. Finally, the spin-echo spectrometer IN11 at the ILL was used to complete these measurements with a better energy resolution $(\sim 0.6-\mu \mathrm{eV}$ resolution) between 150 and $485 \mathrm{~K}$ [a measurement at $2 \mathrm{~K}$ being necessary for the determination of the normalization factor $I(Q, 0)$; see Sec. IV].

The quantity measured in a TOF experiment is the double differential scattering cross section, proportional to the dynamical structure factor $S(Q, \omega) .^{30,31}$ The $S(Q, \omega)$ function corresponds to the space-time Fourier transform of the pair correlation function $G(r, t)$, and represents therefore a physical observable bridging the gap between the microscopic theoretical description and the macroscopic experimental measurements. As $G(r, t)$ contains both "self" and "distinct" contributions, $S(Q, \omega)$ consists of both incoherent and coherent terms,${ }^{30}$ and single-particle and collective contributions could partially overlap. However, in the case of peapods, one can neglect the single-particle incoherent contribution as peapods are namely composed of carbon-which is a purely coherent scatterer. ${ }^{32}$ A spin-echo experiment gives direct access to the intermediate scattering function $I(Q, t)$, which is linked to $S(Q, \omega)$ and $G(r, t)$ by a time or a space-Fourier transform, respectively.

An additional function that we will call "susceptibility"particularly useful for our data analysis and its theoretical interpretation-is defined as the imaginary part of the response function divided by the frequency, $\chi^{\prime \prime}(Q, \omega) / \omega$. The fluctuation-dissipation theorem connects the linear-response theory approach to the pair-correlation function formalism, and in the high-temperature limit of $k_{B} T \gg \hbar \omega$, this theorem can be approximated by $\chi^{\prime \prime}(Q, \omega) / \omega \propto S(Q, \omega) / T$. The generalized phonon density of states $G(Q, \omega)$ is given by ${ }^{33}$ $G(Q, \omega)=\frac{S(Q, \omega)}{Q^{2}} \frac{\omega}{n(\omega, T)}$, where $n(\omega, T)$ is the Bose population. This function is known to be temperature independent for harmonic crystals ${ }^{33}$ since it corresponds to a count of the modes (weighted by the masses and the scattering lengths of the scatterers) in a $d \omega$ interval. In the high-temperature approximation, one thus has $\chi^{\prime \prime}(Q, \omega) / \omega \propto \omega^{-2} G(Q, \omega)$. Therefore, since the susceptibility is temperature independent for a harmonic crystal, it is the most suited function in order to monitor any deviation from a harmonic crystal behavior. All the above listed functions are equivalent and represent the same physics, the choice of using one or another depending only on the focus of the discussion.

\section{THEORETICAL MODEL}

In this section, we calculate the dynamical structure factor $S(Q, \omega)$ for a harmonic chain of $N$ molecules. We first consider the case of a pinned crystalline chain and discuss the elastic and inelastic contributions to the intensity scattered by such a system. In a second step, we discuss the dynamical response of a harmonic liquid chain, which extends the crystalline case to infinite $N$ and/or high temperature. This reminder will help the discussion of the data, which follows in the next part.

\section{A. Reminder about the 1D harmonic chain}

We consider a harmonic 1D lattice of masses $m$ separated by a distance $d$, in which the longitudinal speed of sound is $v$. The Hamiltonian writes ${ }^{34}$

$$
H=\frac{1}{2} \sum_{j=1}^{N}\left[\frac{\pi_{j}^{2}}{m}+\frac{m v^{2}}{d^{2}}\left(u_{j}-u_{j+1}\right)^{2}\right]
$$

with $u_{j}=x_{j}-x_{j}^{0}$ the displacement of the mass indexed $j$ from its equilibrium position $x_{j}^{0}=j d$, and $\pi_{j}=m \dot{u}_{j}$ its momentum. Note that this model only accounts for the longitudinal modes of the chain oriented along the axis $(O x)$.

If $N$ is large enough, edge effects can be neglected and one may apply the Born-von Karman boundary conditions along the axis of the chain. ${ }^{35}$ In that case, the expression of the phonon dispersion equation resulting from the Hamiltonian Eq. (1) is

$$
\omega_{p}=\frac{2 v}{d}\left|\sin \left(q_{p} d / 2\right)\right|, \quad q_{p}=\frac{2 p \pi}{N d}, \quad p=1, \ldots, N,
$$

where $\omega_{p}$ and $q_{p}$ refer to the pulsation and wave vector associated with the $p$ th phonon. In the case of large $N$, 
the associated mean-squared displacement at temperature $T$, $\left\langle u^{2}(T)\right\rangle$, is given by

$$
\begin{aligned}
\left\langle u^{2}(T)\right\rangle & =\frac{1}{N} \sum_{p=1}^{N-1} \frac{\hbar}{2 m \omega_{p}}\left[1+2 n\left(\omega_{p}, T\right)\right] \\
& \simeq \frac{N d^{2} k_{B} T}{2 \pi^{2} m v^{2}},
\end{aligned}
$$

where $n\left(\omega_{p}, T\right)$ is the Bose population of the mode $\omega_{p}$ at temperature $T$, and where the high-temperature approximation is used. Equation (2) shows that for a 1D system, the meansquared displacement $\left\langle u^{2}\right\rangle$ is proportional to the system size $N$, which is a characteristic result of low-dimensional systems. ${ }^{36}$

\section{B. Generalities about neutron scattering}

Let us now consider the intermediate scattering function $I(\boldsymbol{Q}, t)$, which is the space Fourier transform of the paircorrelation function $G(\boldsymbol{r}, t)$ of the observed system- $G(\boldsymbol{r}, t)$ gives the probability to find a scatterer at the position $r$ and at the instant $t$ [i.e., noted $(\boldsymbol{r}, t)]$, if one is at the origin at time $t=0$ [i.e., $(\mathbf{0}, 0)]$. At very long times, correlations become independent of time and one can express $I(\boldsymbol{Q}, t)$ as

$$
I(\boldsymbol{Q}, t)=I(\boldsymbol{Q}, \infty)+I^{\prime}(\boldsymbol{Q}, t) .
$$

Since $S(\boldsymbol{Q}, \omega)$ is the time Fourier transform of $I(\boldsymbol{Q}, t)$, we can write the dynamical structure factor as

$$
S(\boldsymbol{Q}, \omega)=I(\boldsymbol{Q}, \infty) \delta(\omega)+S_{\text {inel }}(\boldsymbol{Q}, \omega) .
$$

In the latter expression, the dynamical structure factor separates into elastic $[I(\boldsymbol{Q}, \infty)]$ and inelastic $\left[S_{\text {inel }}(\boldsymbol{Q}, \omega)\right]$ contributions. We will now calculate these two terms in the case of a $1 \mathrm{D}$ harmonic chain.

\section{Elastic intensity}

The elastic intensity is entirely contained inside $I(Q, \infty)$, i.e., this contribution to the dynamical structure factor characterizes the occurrence of correlations at infinite times. Considering the chain characterized by the Hamiltonian Eq. (1), and writing $\rho(x)$ as the scatterer density along the chain axis, one can write ${ }^{37}$

$$
\begin{aligned}
G(x, t) & =\frac{1}{N} \int d x^{\prime}\left\langle\rho\left(x^{\prime}-x, t=0\right) \rho\left(x^{\prime}, t\right)\right\rangle, \\
I(\boldsymbol{Q}, \infty) & =\frac{1}{N} \int d x e^{-i Q_{x} x} \int d x^{\prime}\left\langle\rho\left(x^{\prime}-x\right)\right\rangle\left\langle\rho\left(x^{\prime}\right)\right\rangle
\end{aligned}
$$

with $Q_{x}$ the projection of the scattering vector $Q$ along the chain axis.

In the case of a harmonic chain of size $N$ with a density $\rho(x)=\sum_{j=1}^{N} \delta\left(x-x_{j}\right)$, this yields

$$
I(Q, \infty)=\frac{1}{N \sqrt{2 \pi}} e^{-\left\langle u^{2}\right\rangle Q_{x}^{2}} \frac{\sin ^{2}\left(Q_{x} d N / 2\right)}{\sin ^{2}\left(Q_{x} d / 2\right)} .
$$

Equation (6) represents the well-known expression of the intensity diffracted by a crystalline chain of size $N$, the thermal fluctuations of the atoms being accounted for by the presence of the Debye-Waller factor $e^{-\left\langle u^{2}\right\rangle} Q_{x}^{2}$. The powder averaging of this expression results in the characteristic $Q$-asymmetric feature extensively developed in Refs. 38 and 27. Close to

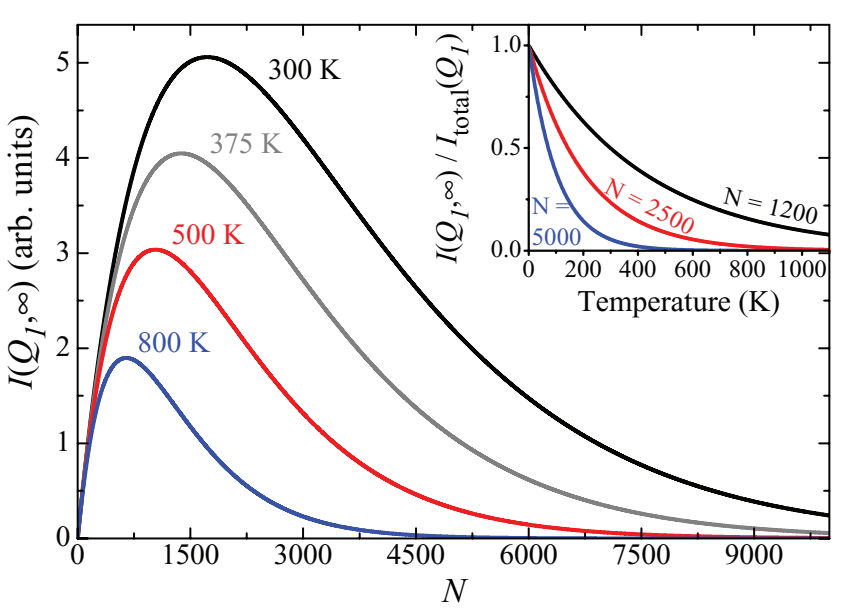

FIG. 2. (Color online) Evolution of $I\left(Q_{1}, \infty\right)$ as a function of the system size $N$ and of the temperature, for a harmonic chain of $\mathrm{C}_{60}$ with speed of sound $v=3.5 \mathrm{~km} \mathrm{~s}^{-1}$ (value determined from our experiments; see Sec. IV). The inset shows the evolution of $I\left(Q_{1}, \infty\right) / I_{\text {total }}\left(Q_{1}\right)$ as a function of temperature for various chain sizes.

$Q=Q_{1}=2 \pi / d$, the squared ratio of the sine functions tends towards $N^{2}$, whereas $\left\langle u^{2}\right\rangle$ is linear with $N$ as shown in Eq. (2): the scattered intensity is thus governed by the competition between an increasing number of scatterers and increasing thermal vibrations.

The evolution of the elastic intensity is represented in Fig. 2. This figure shows that above a certain chain size, the elastic contribution decreases when the temperature or the number of molecules increases. This means that a chain smaller than a few thousand constituents will behave as a harmonic crystal at room temperature-i.e., correlations remain at long (infinite) times. As the number of molecules increases, the chains progressively evolve to a liquid state-i.e., the correlations are lost at long (infinite) times. This results in the vanishing of the elastic contribution of the scattering. This difference in behavior is explained qualitatively by the $N$ dependence of the mean-squared displacement: for small systems, the molecules remain well localized around their equilibrium positions, and the behavior is that of a crystal. For very large systems, the molecules will explore an area eventually as broad as the inter- $\mathrm{C}_{60}$ distance and behave like a liquid. Such a liquid character for very long atomic harmonic chains has, for instance, been observed in various composite crystals. ${ }^{36,39-41}$ A well-known phenomenological argument-the Lindemann threshold $^{42}$ - states that a crystal has melted when the atomic displacements have reached between 15 and 30\% (depending on the system) of the crystalline interatomic distance. In most common systems, such a limit is reached when the anharmonic terms of the potential become important. In a 1D chain, however, this limit can also be reached for a purely harmonic potential.

Given the above discussion, two limit cases are to be considered: the case where the number $N$ of $\mathrm{C}_{60}$ molecules per chain and the associated $\left\langle u^{2}\right\rangle$ are very large (i.e., the liquid case), and the one where $N$ is small enough so that $\left\langle u^{2}\right\rangle$ remains small compared to $d^{2}$ (i.e., the crystal case). One shall emphasis here is that these two cases are but limited cases: 
there is no "phase transition" in the usual sense between the two cases (as expected for a large 1D system with short-range interactions ${ }^{43}$ ), but rather a progressive evolution from one limit to the other, as is illustrated by Fig. 2 and its inset.

\section{Inelastic intensity}

In the following, we calculate the coherent dynamical structure factor for $1 \mathrm{D}$ chains of rigid $\mathrm{C}_{60}$ molecules described by the Hamiltonian Eq. (1). The rigid body approximation appears reasonable as the internal modes of the molecules are well separated from that of the external modes (a gap of $20 \mathrm{meV}$ exists between the lattice modes and the first molecular vibration in the $\mathrm{C}_{60}$ crystal $^{19}$ ). Therefore, the only degree of freedom in this model concerns the longitudinal translations of the molecules' center of mass. The justification of ignoring the rotational and transverse acoustic modes will appear clearly in the next section.

\section{Crystal case}

Let us recall that we have defined the crystal case by the case where the system size $N$ is finite. This results in a small $\left\langle u^{2}\right\rangle$ with respect to $d^{2}$, which in turn results in the occurrence of structural correlations at infinite times (translated in the INS by the presence of an elastic intensity around $Q=Q_{1}$ )—which is characteristic of a crystal.

In the $1 \mathrm{D}$ system we have considered until now and in the case where only the one-phonon events are accounted for, it can be shown that the inelastic part of the dynamical structure factor takes the form ${ }^{30,37}$

$$
\begin{aligned}
S_{\text {inel }}(\boldsymbol{Q}, \omega)= & \frac{\sigma_{C}^{\mathrm{coh}}}{2 d m} \sum_{l} \sum_{\left\{q_{p}\right\} \in \mathrm{BZ}} \frac{\left|F\left(\boldsymbol{Q} \mid q_{p}\right)\right|^{2}}{\omega_{p}} \\
& \times\left\{\left[1+n\left(\omega_{p}\right)\right] \delta\left[q_{p}-\left(Q_{x}+Q_{l}\right)\right] \delta\left(\omega-\omega_{p}\right)\right. \\
& \left.+n\left(\omega_{p}\right) \delta\left[q_{p}+\left(Q_{x}+Q_{l}\right)\right] \delta\left(\omega+\omega_{p}\right)\right\}
\end{aligned}
$$

with $Q_{l}=\frac{2 \pi l}{d}(l=1, \ldots, N)$ the reciprocal-lattice vectors, $\sigma_{C}^{c o h}$ the coherent cross section of a carbon atom, BZ the first Brillouin zone (i.e., $q_{p}=\frac{2 p \pi}{N d}$ with $p=1, \ldots, N$ ), and $F\left(\boldsymbol{Q} \mid q_{p}\right)$ the phonon form factor. In the rigid body approximation, the phonon form factor writes

$$
\left|F\left(\boldsymbol{Q} \mid q_{p}\right)\right|^{2}=|F(Q)|^{2} Q_{x}^{2} .
$$

Equation (8) implies that a longitudinal component of the scattering vector $\boldsymbol{Q}$ is necessary to observe the longitudinalacoustic modes. If $\boldsymbol{Q}$ is set orthogonal to the chain axis (i.e., $Q_{x}=0$ ), the scattered intensity is null.

In the following, we will focus on $l=1$ and will therefore only consider acoustic modes at low wave vectors (smaller than $2 \AA^{-1}$ ), which results in approximating the $\mathrm{C}_{60}$ molecules by homogeneous spheres. In that case, the $\mathrm{C}_{60}$ form factor is given by $F(Q)=60 \operatorname{sinc}\left(Q r_{C_{60}}\right)$, with $r_{C_{60}}=3.55 \AA$ the radius of a $\mathrm{C}_{60}$ molecule. ${ }^{27}$ This form factor is at the origin of the intensity difference of the chain features observed in the diffraction patterns of the monomer and polymer peapods, Fig. 1, $F(Q)$ being a decreasing function in the $[0,0.88] \AA^{-1} Q$ range. In addition we use the high-temperature approximation, and we suppose that $N$ is large enough to use the integral limit of the above expressions.

In the case where $Q$ is set parallel to the chain axis ( $Q_{x}=$ $Q$ ), Eq. (7) writes

$$
\begin{aligned}
S_{\text {inel }}(Q, \omega)= & \frac{\sigma_{C}^{\text {coh }} N d^{2} k_{B} T}{8 h m v^{2}}|F(Q)|^{2} \frac{Q^{2}}{|\sin (Q d / 2)|^{2}} \\
& \times\left[\delta\left(\omega+\frac{2 v}{d}|\sin (Q d / 2)|\right)\right. \\
& \left.+\delta\left(\omega-\frac{2 v}{d}|\sin (Q d / 2)|\right)\right] .
\end{aligned}
$$

One shall note here that Eq. (9) shows that in the case of a 1D harmonic crystal, $S_{\text {inel }}(Q, \omega) / T \propto \chi^{\prime \prime}(Q, \omega) / \omega$ is, as expected, independent of temperature.

The convolution of Eq. (9) with an experimental energy resolution is shown in Fig. 3(a). One observes the dispersion branch of a single-crystalline fiber that would be experimentally obtained keeping $\boldsymbol{Q}$ parallel to the chains axis at each $(Q, \omega)$. Note that the extinction observed at around $Q=0.88 \AA^{-1}$ is due to the fullerene form factor $F(Q)$ which goes to zero at this value.

When taking into account a Gaussian off-plane orientation distribution and the specific TOF geometry, the 2D powder averaging of the dynamical structure factor results in the scattering map shown in Fig. 3(b). ${ }^{28}$ For these calculations, an off-plane misalignment of $30^{\circ}$ half width at half maximum (HWHM) was injected into the calculations. This value was determined by former diffraction investigations. ${ }^{28}$ The specific response from the longitudinal acoustic phonon takes the form of a triangular-shaped signal located in the vicinity of the elastic line at the first $\mathrm{C}_{60}$ correlation peak (i.e., at $\left.Q_{1}=2 \pi / d \simeq 0.64 \AA^{-1}\right)$.

Consider the function $S(\omega)$ [respectively $\chi^{\prime \prime}(\omega / \omega)$ ], defined as the $Q$ integration of $S(Q, \omega)$ [respectively $\chi^{\prime \prime}(Q, \omega / \omega)$ ] in the $[0.5,0.8] \AA^{-1}$ range, where the signal from the first-order peak is located. By definition,

$$
S(Q, \omega) \propto\left(\frac{\operatorname{sinc}\left(Q r_{C_{60}}\right)}{\operatorname{sinc}(Q d / 2)}\right)^{2} S(\omega) .
$$

Besides, $\frac{\omega^{2}}{T} S(\omega)$ is proportional to the density of state of the acoustic phonon $G(\omega)$ which, in the long-wavelength limit, is given by $G(\omega)=1 / v$. Consider now two chains of $\mathrm{C}_{60}$ with two different speeds of sound, $v$ and $v^{\prime}$, and two different lattice parameters, $d$ and $d^{\prime}$ (e.g., monomer and polymer peapods). If the $Q$ integration of the dynamical structure factors $S(Q, \omega, v, d)$ and $S\left(Q, \omega, v^{\prime}, d^{\prime}\right)$ are performed over a sufficiently large range around $2 \pi / d$ and $2 \pi / d^{\prime}$, one has the following relationship:

$$
S\left(\omega, v^{\prime}, d^{\prime}\right)=\eta \eta_{v} \times S(\omega, v, d)
$$

with $\eta$ being given by

$$
\eta=\left(\frac{\operatorname{sinc}\left(2 \pi r_{C_{60}} / d\right)}{\operatorname{sinc}\left(2 \pi r_{C_{60}} / d^{\prime}\right)}\right)^{2}
$$

and

$$
\eta_{v}=\frac{v}{v^{\prime}}
$$



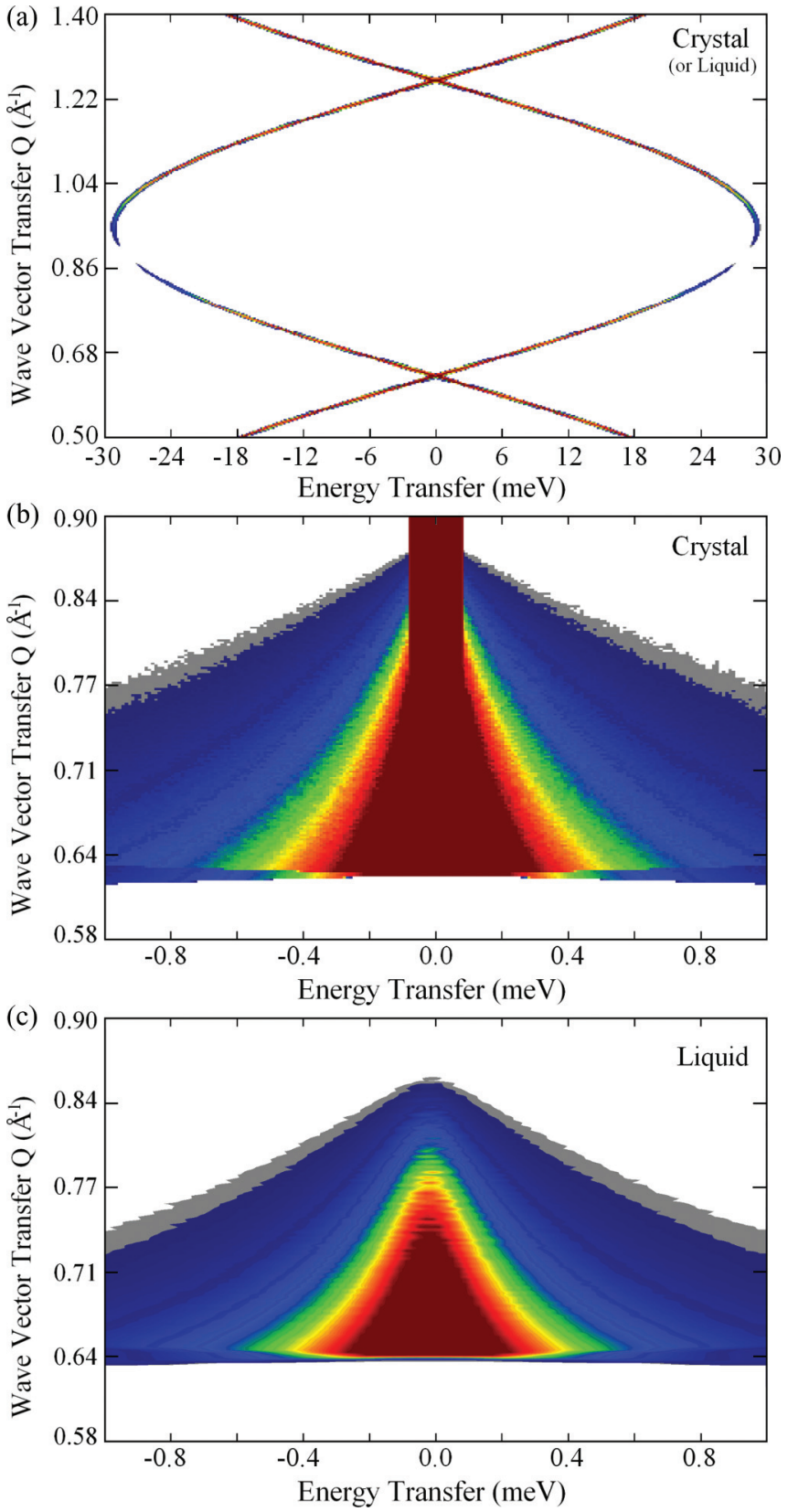

FIG. 3. (Color online) Simulated inelastic part of the dynamical structure factor $S(Q, \omega)$ scattered by harmonic $\mathrm{C}_{60}$ chains, with $d=$ $10 \AA, v=3.5 \mathrm{~km} \mathrm{~s}^{-1}$ and a Gaussian energy resolution of FWHM $=$ $25 \mu \mathrm{eV}$ : (a) for a fiber of crystalline $\mathrm{C}_{60}$ chains and with $\boldsymbol{Q}$ collinear to the fiber at each $(Q, \omega)$ point; (b) on a TOF spectrometer for a pellet of crystalline $\mathrm{C}_{60}$ chains with off-plane orientations distribution of $\pm 30^{\circ}$. The combined effect of the $2 \mathrm{D}$ anisotropy of the sample and of the TOF geometry is responsible for the triangular shape of the observed $S(Q, \omega)$ signal-whose intensity is proportional to the density of states of longitudinal-acoustic phonons. The intensity is saturated for a better viewing, the central line being due to elastic scattering; (c) on a TOF spectrometer for a pellet of liquid $\mathrm{C}_{60}$ chains (infinite chains). In that case, the intensity is of purely inelastic nature.

Therefore, if both monomer and polymer peapods behave like $1 \mathrm{D}$ harmonic crystals, their $Q$-integrated dynamical structure factors (respectively susceptibilities) should only differ by a factor $\eta \eta_{v}$ with $\eta_{v}=v_{\text {mono }} / v_{\text {poly }}$.

\section{Liquid case}

We have defined the liquid case as a $1 \mathrm{D}$ harmonic chain where the number of constituent $N$ is very large. This results in a large $\left\langle u^{2}\right\rangle$ with respect to $d^{2}$ and in the loss of structural correlations at long times. This translates into the INS spectra by the absence of elastic intensity around $Q=Q_{1}$, which is characteristic of a liquid.

In such a model, the mean-squared displacement becomes too large to neglect the multiphonon terms. It can be shown that the dynamical structure factor takes the form ${ }^{34,36,40}$

$$
S(Q, \omega) \propto \frac{\alpha_{l}^{2}|F(\boldsymbol{Q})|^{2}}{\left(\alpha_{l}^{2}+\left(q_{l}+\frac{\omega}{v}\right)^{2}\right)\left(\alpha_{l}^{2}+\left(q_{l}-\frac{\omega}{v}\right)^{2}\right)},
$$

where $\alpha_{l}=2 l^{2} \pi^{2} k_{B} T / d m v^{2}$ and $q_{l}=Q-2 l \pi / d$. In the derivation of this expression, and in contrast to the previous case, no separation between elastic and inelastic scattering has been made. ${ }^{28,40}$ Therefore, the signal scattered by an infinite harmonic chain is of purely inelastic nature, which is characteristic of a liquid. The resulting map is shown in Fig. 3(c). The triangular feature already discussed in the crystalline case is also observed, the difference between the two cases residing in the absence of an elastic contribution in the liquid spectrum.

When integrated over $Q$, Eq. (12) yields (since only the $T$ dependence is investigated, we neglect the molecule's form factor)

$$
S(\omega) \propto \frac{\alpha_{l}}{\left(\frac{\omega}{v}\right)^{2}+\alpha_{l}^{2}} .
$$

As a consequence, if the confined $\mathrm{C}_{60}$ chains were to behave as harmonic liquid chains, the longitudinal acoustic-phonon signal would result in a purely Lorentzian quasielasticlike signal located around $Q_{l}=2 \pi l / d$, whose width would vary linearly with $T / v^{2}$. The fact that the width of the signal evolves with temperature even in the harmonic hypothesis results from the damping of the acoustic phonons via multiphonon interaction.

Let us summarize the principal information one can extract from these two models. In the case of chains of "reasonable size" at sufficiently "low" temperatures, one expects a crystalline behavior, which would be translated into the dynamical structure factor by (i) an elastic contribution, and (ii) an inelastic contribution whose width does not evolve with temperature. In the case of very long chains or high temperature, the expected behavior is that of a liquid, which will translate in the INS spectrum by the absence of an elastic contribution and a signal whose energy broadening should evolve as $T / v^{2}$

Finally, one shall emphasize that the coherent signal in Figs. 3(b) and 3(c) can be labeled as "quasielastic" because of its location around the elastic line. Nevertheless, in both crystal and liquid cases, this signal arises from the density of states of longitudinal-acoustic phonons and therefore does not correspond to any diffusive process.

\section{RESULTS AND DISCUSSION}

\section{A. Isolating the longitudinal modes}

The experimental strategy followed during the neutron investigations resides on isolating the longitudinal modes of the 
confined chains by combining the 2D anisotropy of the peapod pellets, and on the strong dependence of the scattered intensity with the orientation of the scattering vector $\boldsymbol{Q}$ with regards to the chain axis [Eq. (8)]. Therefore, two measurements were performed at each temperature. They are referred to as "para" and "ortho" in the following, and correspond to configurations in which the plane of the sample holder is set either parallel or orthogonal to the elastic-scattering vector $Q$ with $Q=Q_{1}$. According to Eq. (8), the signal arising from the longitudinal translations of the confined $\mathrm{C}_{60}$ molecules will be enhanced in the para configuration compared to the ortho one. Using a specific data treatment scheme presented in the Appendix, we managed to isolate the longitudinal response of the $\mathrm{C}_{60}$ chains in the TOF data. This treatment is based on the hypothesis that the inelastic scattering arising from the nanotubes is weak and not strongly dependent on the sample orientation in the $(Q, \omega)$ region of interest. This assumption appears rather reasonable as the dynamics of the nanotube bundles consists essentially of internal "radial" modes involving atomic displacements perpendicular to the tube axis. Therefore the dot product in Eq. (8) should not vary for these modes between the two configurations, and the tubes contribution is eliminated by a "controlled" subtraction (see the Appendix).

After data manipulation, the longitudinal contribution of the chain can be isolated and one obtains the typical triangular feature discussed in the previous sections. An illustration of the data treatment is presented in Fig. 4 showing the $S(Q, \omega)$ maps taken on IN5 at $420 \mathrm{~K}$ in both para and ortho configurations. Let us note that the fixed geometry of a TOF instrument implies that the sample plane will only be parallel (respectively orthogonal) to the wave vector $\boldsymbol{Q}$ for $Q=Q_{1}$ and $\omega=0$. For example, in the vicinity of the second "diffraction" order at $Q_{2} \simeq 1.28 \AA^{-1}$, the angle between $\boldsymbol{Q}$ and the sample is around $\sim 30^{\circ}$ in both configurations. Due to the $\pm 30^{\circ}$ off-plane distribution of the orientations of the bundles, the scattering function remains almost configuration independent around $Q_{2}$. Therefore when discussing the TOF data, we will restrict ourselves to the region where $Q$ belongs to $[0.5,0.8] \AA^{-1}$ and $\omega$ stays within $[-0.5,0.5] \mathrm{meV}$ in order to monitor the most parallel and the most orthogonal configurations. In addition, as far as the polymer phase of the peapods is concerned, $S(\omega)$ and the derived susceptibility are further corrected for the factor $\eta$ discussed in Eq. (11) ( $d$ and $d^{\prime}$ being the 1D lattice parameters of the monomer and polymer phases, respectively).

Figure 5(a) shows the normalized intermediate structure factor $I(Q, t) / I(Q, 0)$ for different $Q$ values and different temperatures $T$ obtained using the spin-echo spectrometer IN11. The instrument geometry is such that for every $Q$ value, $\boldsymbol{Q}$ is set either parallel or orthogonal to the scattering plane. Each data set was fitted using the function

$$
\frac{I(Q, t)}{I(Q, 0)}=I(Q, \infty)+I_{\text {inel }}(Q) e^{-t / \tau},
$$

which allowed deriving the $Q$ and $T$ dependence of the "elastic" $I(Q, \infty)$ and "inelastic" $I_{\text {inel }}(Q)$ contributions of the scattering. For each $Q$ in the para configuration, the data can be reproduced keeping a constant "relaxation time" $\tau_{\text {para }}$ over the complete temperature range of the experiment. The evolutions of $I_{\text {inel }}(Q)$ and $\tau_{\text {para }}$ with $Q$ for a temperature $T=485 \mathrm{~K}$ are shown Fig. 5(b) for both ortho and para
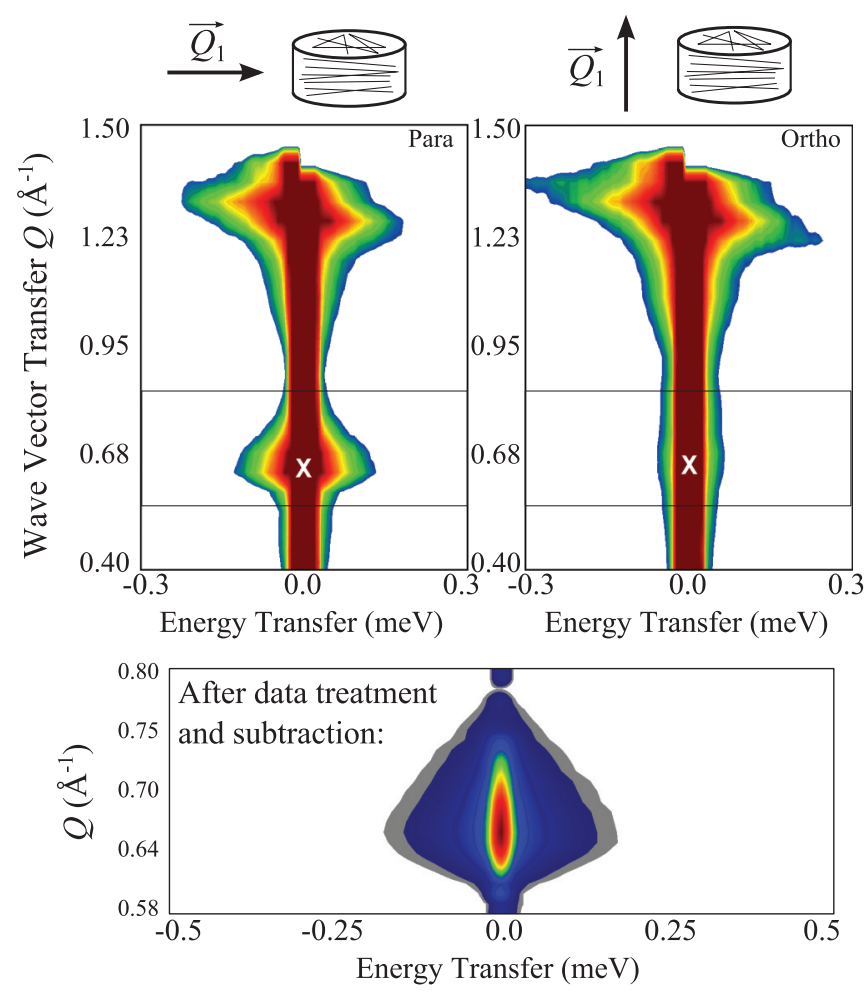

FIG. 4. (Color online) Top: $S(Q, \omega)$ maps taken at $420 \mathrm{~K}$ on IN5, for incident wavelength of $8 \AA$, in the parallel (left) and orthogonal (right) configurations. Maximum intensity is saturated for clarity. The white cross indicates the point $\left(Q=Q_{1}, \omega=0\right)$, the only point where the wave vector transfer $\boldsymbol{Q}$ with a norm $Q_{1}$ is strictly either parallel or orthogonal to the pellet. The horizontal lines define the $Q$ range considered in the integration to obtain $S(\omega)$ [see Fig. 6(a)]. Bottom: $S(Q, \omega)$ map at $485 \mathrm{~K}$ after data treatment and subtraction (description in the Appendix).

configurations. By lack of time during the experiment, the ortho configuration was measured only around the second order $Q_{2}$. The anisotropy of $I_{\text {inel }}(Q)$ for $Q \sim Q_{2}$ is attributed to the signature of the longitudinal motions of the $\mathrm{C}_{60}$ molecules, and the same anisotropy is expected around $Q_{1}$.

\section{B. "Low" temperatures}

The TOF $Q$-integrated dynamical structure factor $S(\omega)$ obtained at $373 \mathrm{~K}$ is shown in Fig. 6(a). It is very well reproduced by a scattering function modeled by an elastic contribution added to a Lorentzian line shape to account for low-frequency inelastic scattering. The $\sim 60 \mu \mathrm{eV}$ HWHM of the inelastic contribution agrees well with the "relaxation time" $\tau \sim 50 \mathrm{ps}$ derived from the spin-echo data at the same $Q$ values. ${ }^{45}$ These observations suggest that the signal observed on both instruments arises from the same dynamics.

The temperature evolution of the susceptibility $\chi^{\prime \prime}(\omega) / \omega$ in the temperature range $[260,420] \mathrm{K}$ derived from the TOF data is represented on Fig. 6(b) for the monomer and polymer phases of the peapods. The susceptibilities are found to be constant in this temperature range for both peapod phases. In addition, the inset of Fig. 5(a) shows the temperature evolution of the inelastic part of the spin-echo signal $I_{\text {inel }}(Q)$ measured at $Q=Q_{1}$ on IN11. The linear temperature dependence of the 


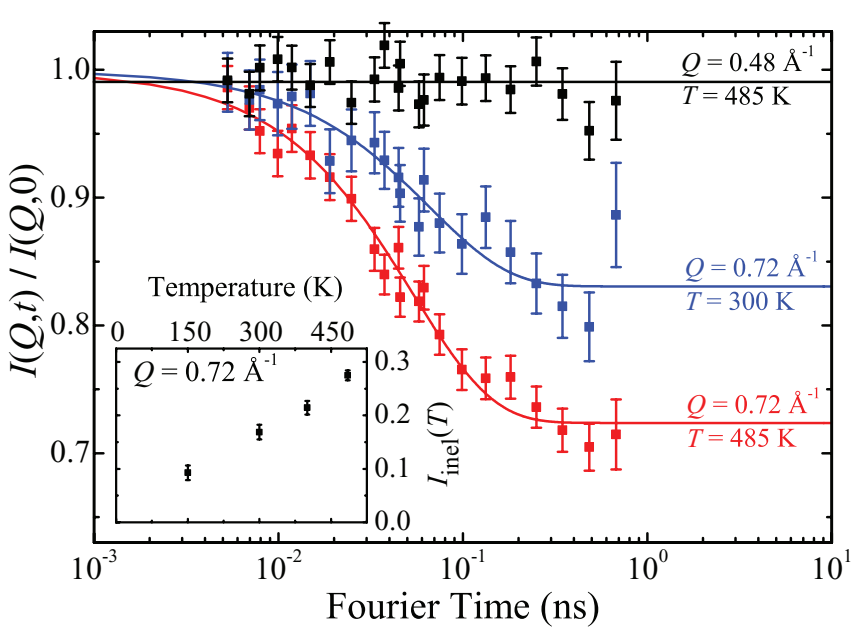

(a)

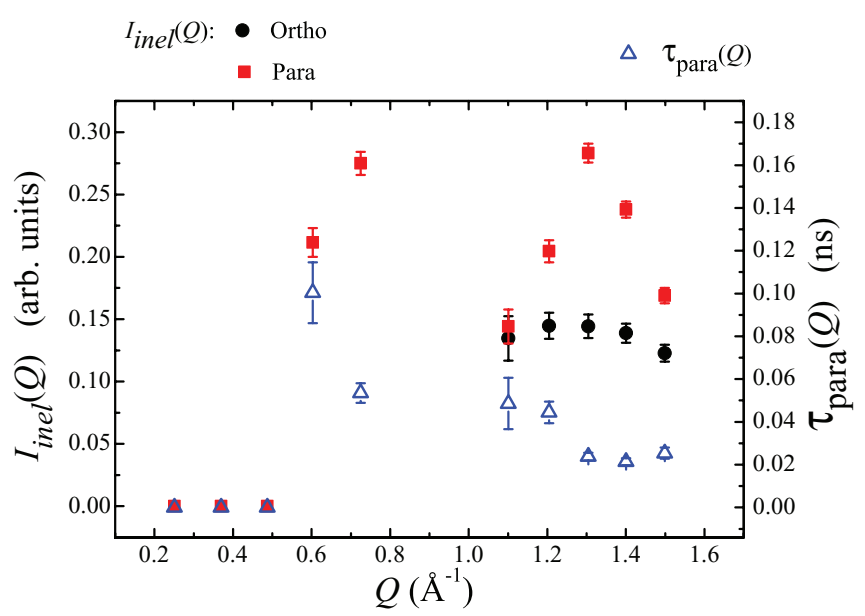

(b)

FIG. 5. (Color online) (a) Normalized intermediate scattering function $I(Q, t) / I(Q, 0)$ for $Q=0.48 \AA^{-1}$ and $Q=0.72 \AA^{-1}$ at different temperatures, as measured in the parallel configuration on the spin-echo spectrometer IN11 at the ILL. Data are fitted by a decreasing exponential $I(Q, \infty)+I_{\text {inel }} e^{-t / \tau}$ (full lines). The inset shows the temperature evolution of $I_{\text {inel }}$ for $Q=0.72 \AA^{-1}$ in the para configuration, fitted with a constant characteristic time $\tau=53$ ps. (b) Evolution of the quasielastic intensity $I_{\text {inel }}$ (in both para and ortho configurations) and of the characteristic time $\tau_{\text {para }}$ as a function of $Q$ at $485 \mathrm{~K}$. The additional intensity in the parallel configuration around $Q=Q_{2} \simeq 1.3 \AA^{-1}$ confirms that the observed dynamics concerns longitudinal motions along the $\mathrm{C}_{60}$ chains.

intensity of $I_{\text {inel }}(Q)$, associated with the constant evolution of the susceptibility and the presence of an elastic contribution in $S(\omega)$ reveal (i) the crystalline behavior of the chains and (ii) the harmonic nature of the longitudinal excitations measured, which we associate to the longitudinal-acoustic phonons of the $\mathrm{C}_{60}$ chains. In this temperature range, the confined $\mathrm{C}_{60}$ chain can therefore be completely described by a classical 1D harmonic model of finite length, which is accounted for by the Hamiltonian Eq. (1). In particular, no sign of any slow diffusion process is detected. Such processes could originate from $\mathrm{C}_{60}$ cluster (or void) diffusion along the tube.

Figure 6(b) shows that the polymer susceptibility at $320 \mathrm{~K}$ superposes to that of the monomer after applying a scaling

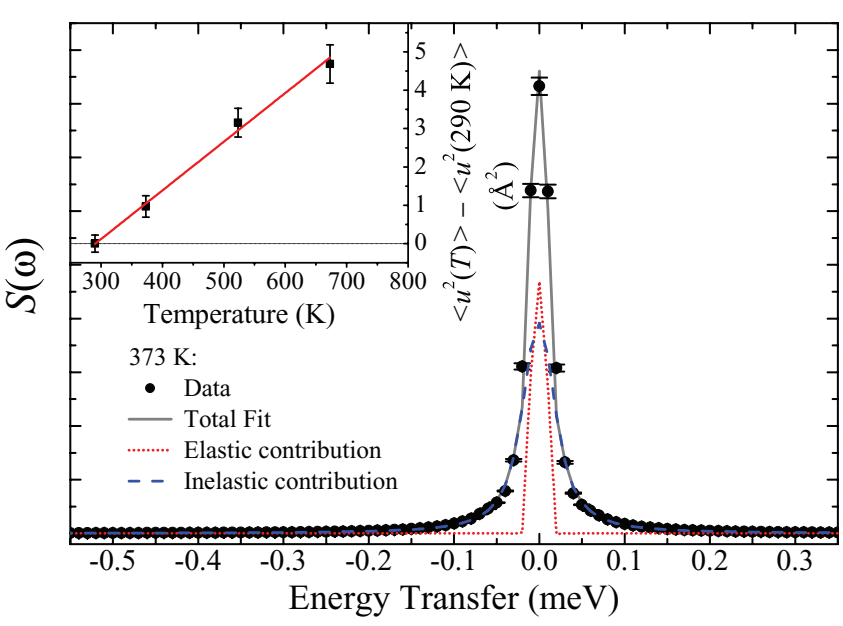

(a)

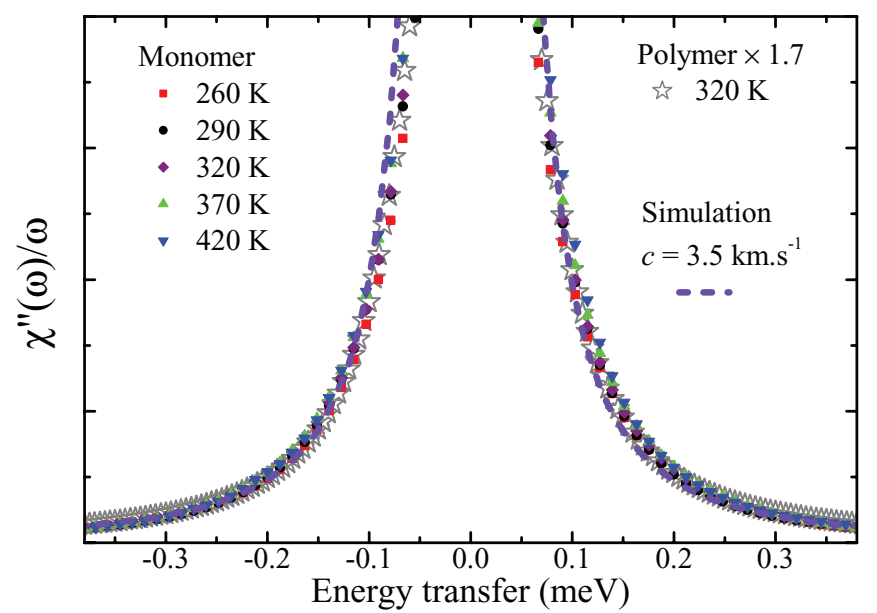

(b)

FIG. 6. (Color online) (a) Dynamical structure factor $S(\omega)$ at $373 \mathrm{~K}$ as measured on TOFTOF with incoming wavelength of $8 \AA$. These are the data after full treatment, data subtraction, and $Q$ integration over the range [0.5,0.8] $\AA^{-1}$. Data are fitted using a Dirac function convoluted with experimental resolution (elastic) and a Lorentzian function (inelastic). The inset shows the temperature evolution of $\left\langle u^{2}(T)\right\rangle-\left\langle u^{2}(290 \mathrm{~K})\right\rangle$ and its linear fit (see text). (b) Temperature evolution of the susceptibility $\chi^{\prime \prime}(\omega) / \omega$ of the quasielastic signal between 260 and $420 \mathrm{~K}$ as measured on IN5 for monomer peapods, and susceptibility from the polymer $\mathrm{C}_{60}$ peapod at $320 \mathrm{~K}$ (empty stars), normalized to the $\mathrm{C}_{60}$ masses ratio within the two samples, to the $\eta$ coefficient, and multiplied by 1.7. The dashed line corresponds to a simulation for a sound velocity of $3.5 \mathrm{~km} \mathrm{~s}^{-1}$ and an angular off-plane distribution of $\pm 30^{\circ}$. Error bars are not represented for the sake of clarity.

factor $\eta_{v}=1.7$ [the data are already corrected for the factor $\eta$ in Eq. (11)]. The large difference in the attractive strength between covalent bonds and van der Waals interactions is such that it is reasonable to expect that the speed of sound in polymerized peapods is similar to that in orthorhombic bulk $\mathrm{C}_{60}$, where the fullerenes form $1 \mathrm{D}$ polymerized chains. The speed of sound along these polymer chains was determined to $\mathrm{be}^{46} v_{\text {poly }}=6.0 \mathrm{~km} \mathrm{~s}^{-1}$. Using Eq. (11), this enables determination of the speed of sound in the $\mathrm{C}_{60}$ chains present in the monomer peapods: we obtain $v_{\text {mono }} \simeq 3.5 \pm 0.25 \mathrm{~km} \mathrm{~s}^{-1}$. This value is found to be close to the $\langle 110\rangle$ direction speed 
of sound in bulk $\mathrm{C}_{60}$, which was measured to be ${ }^{46} v_{\langle 110\rangle}=$ $3.35 \mathrm{~km} \mathrm{~s}^{-1}$.

Taking this value for the speed of sound, it is further possible to extrapolate the mean chain size of the monomer $\mathrm{C}_{60}$ chain from the temperature evolution of the Debye-Waller factor. The latter quantity is derived from the evolution of the elastic intensity given by Eq. (6). The inset of Fig. 6(a) shows the evolution of $\left\langle u^{2}(T)\right\rangle-\left\langle u^{2}(290 \mathrm{~K})\right\rangle$, with $\left\langle u^{2}(T)\right\rangle$ given by Eq. (2). The slope of the linear fit of these data allows extracting the quantity $N d^{2} k_{B} / 2 \pi^{2} m v^{2}$, from which we can evaluate $N \simeq 2700$. This rather long chain size is perfectly compatible with the high filling ratio (97\%) deduced from our x-ray scattering characterizations ${ }^{21}$ and with the average size of the nanotubes estimated by TEM, i.e., between 1 and $5 \mu \mathrm{m} .^{23}$

Knowing $v$ and $N$ and injecting them into Eq. (2), the value of $\left\langle u^{2}(T)\right\rangle$ is found to be $3.7 \AA^{2}$ at $290 \mathrm{~K}$ and $7.7 \AA^{2}$ at $600 \mathrm{~K}$. Therefore, taking a Lindemann threshold of $30 \%$ of the lattice parameter, one anticipates that the chains should fully behave as a liquid around the temperature of $\sim 700 \mathrm{~K}$.

\section{Melting and liquid phase}

Figure 7(b) represents the temperature evolution of the proportion of the elastic intensity with respect to the total intensity as well as the evolution of the inelastic intensity, as derived from the TOF experiments for monomer peapods [intensities are obtained from the integration of the different contributions of the fitted data; see Fig. 6(a)]. One observes a progressive disappearance of the elastic line with increasing temperature, with total disappearance for $T \gtrsim 850 \mathrm{~K}$. The inelastic part of the scattered intensity is linear with $T$ up to $\sim 600 \mathrm{~K}$ where it progressively evolves to a constant value. Even though no discontinuity is observed in the evolutions, the behaviors at the extreme opposite sides of the temperature range are very different. As already discussed, the dynamics below $\sim 550 \mathrm{~K}$ is well understood using a harmonic crystal picture. The absence of elastic intensity above $T \gtrsim 850 \mathrm{~K}$ translates the disappearance of structural correlations at long time, e.g., a liquid behavior, the scattered intensity becoming purely dynamic in nature, as discussed in Sec. IIID2. The intensity of the inelastic signal is found to be temperature independent in this range. If we invoke the quasiharmonic approximation, the liquid phase at high temperature can be modelled using the harmonic Hamiltonian (1) in the high-temperature limit. The dynamical structure factor takes the form (13), and the speed of sound in the liquid phase $v_{l}$ is related to the FWHM of the Lorentzian line shape of the inelastic signal. We find a value of $v_{l} \sim 1.8 \mathrm{~km} \mathrm{~s}^{-1}$ at $1040 \mathrm{~K}$. This value is $51 \%$ smaller than in the crystalline phase, and is observed to be almost constant in the $T$ range [823,1043] K. This important change in the sound velocity translates the softening of the elastic constants when the 1D solid melts, and is found to be equivalent - in amplitude - to the melting transition between ice and water. The transformation occurs gradually in a $T$ range of $\sim 150 \mathrm{~K}$ centered around $600 \mathrm{~K}$ [Fig. 7(b)]. In a previous study, ${ }^{21}$ we also observed an important change in the linear thermal-expansion coefficient at this temperature. The transformation of the chain's physical nature is clearly revealed by the dynamics, the $1 \mathrm{D}$ order becoming unstable

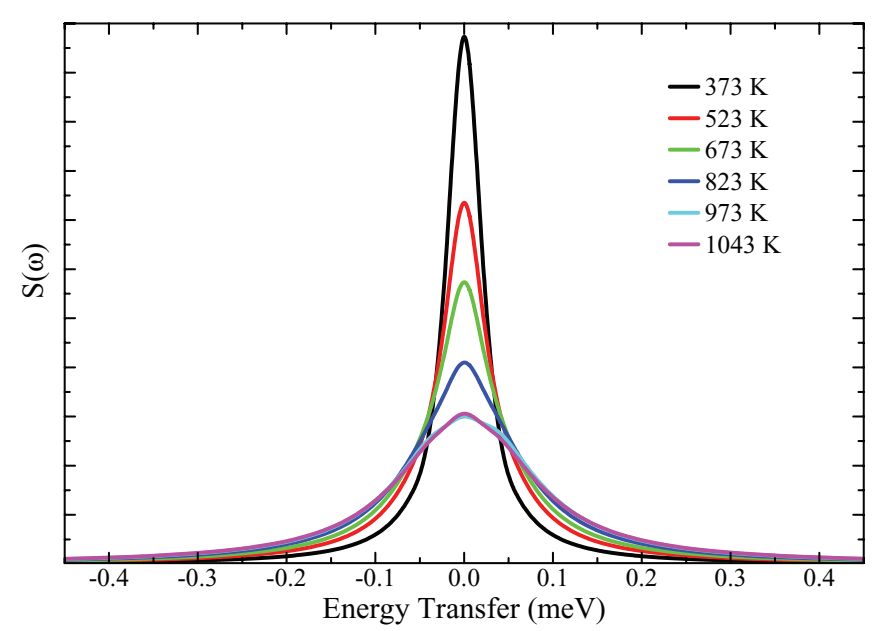

(a)

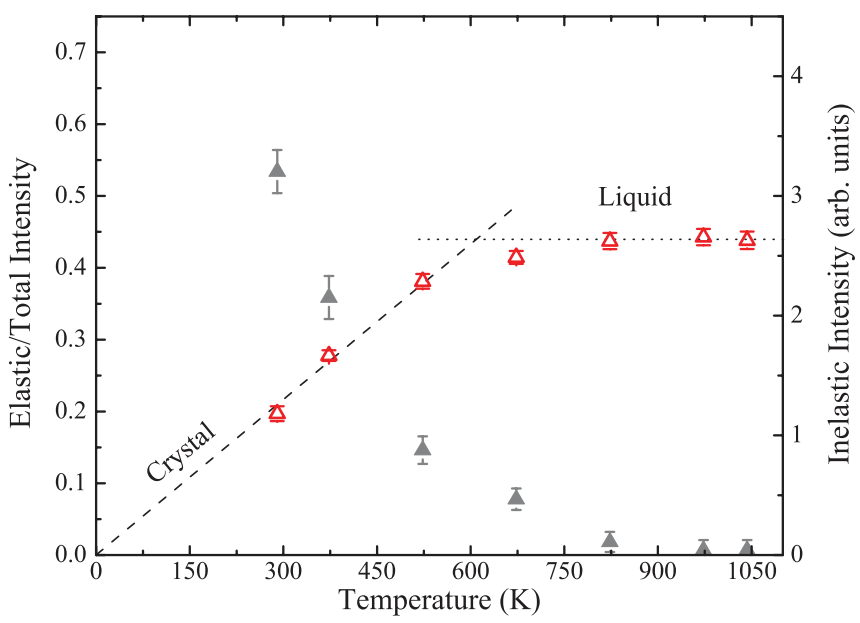

(b)

FIG. 7. (Color online) (a) Temperature evolution of the dynamical structure factor $S(\omega)$ between 373 and $1043 \mathrm{~K}$ as measured on TOFTOF after full treatment of the data. (b) Temperature evolution of the ratio $I_{\text {elastic }} / I_{\text {total }}=I_{\text {elastic }} /\left(I_{\text {elastic }}+I_{\text {inelastic }}\right)$ (full triangles) and of the inelastic intensity (empty triangles) as measured on TOFTOF (intensities are integration of fitted function Fig. 6(a)). Dotted and dashed lines are guides to the eye.

under the fluctuations of the lattice parameter. Moreover, the strong difference between the sound velocities emphasizes that the driving force of the melting has to account for the anharmonicity of the potential, e.g., that the actual scenario is different from what occurs in a very long harmonic chain, as discussed in Sec. III C, for which there is no change in the elastic properties of the chain between the solid and the liquid phase. In our system, at sufficiently high temperatures, the harmonic approximation becomes less and less valid as the full $\mathrm{C}_{60}-\mathrm{C}_{60}$ interaction potential is felt, and the melting process accelerates. The nuance is somewhat subtle, since in both cases it is the large increase of the thermal vibrations and their propagation that lead to a complete loss of the correlations above approximatively $600 \mathrm{~K}$, the resulting structure having the characteristics of a $1 \mathrm{D}$ liquid.

These results-obtained by probing directly the translational dynamics of the confined $\mathrm{C}_{60}$-are coherent with 
those derived by following the temperature dependence of the structure using $\mathrm{x}$-ray diffraction and by its description using a Takahasi-Gû́rsey (TG) model based on a Lennard-Jones intermolecular potential. ${ }^{21}$ The transition between the two limit states of the chain is clearly visible in the temperature evolution of the expansion coefficient which shows a classical evolution up to a temperature at which it vanishes. The set of structural and dynamical data allows us to propose the following scenario. At low temperature, the amplitude of the vibrations is small and the potential appears as harmonic. As the temperature increases, the amplitude of the vibrations becomes large and the anharmonic terms of the intermolecular potential become important. This results in a softening of the vibrations which further accelerates the increase of the molecular displacements. The strong anharmonicity of the interaction potential at high temperatures is illustrated by both the strong asymmetry of the first neighbor distribution function (Fig. 1 of Ref. 21) and the strong reduction of the speed of sound observed in this study. At the highest temperatures, it was suggested that the rigid nanotube host reduces the linear thermal expansion of the chain by limiting its extension. ${ }^{21}$ Limiting the size of the chain while increasing the temperature creates an internal pressure, which should result in an increasing sound velocity at higher temperature. The observation of a constant FWHM of the scattered signal at 973 and $1043 \mathrm{~K}$ is an indication in this direction.

In addition to our previous investigation, the use of INS has allowed us to emphasize the importance of the limited size of the system regarding to its dynamical behavior. This was made possible by discerning elastic from inelastic contributions to the scattering. It is important to emphasize that-again - the model developed in this study does not account for any longitudinal coupling between the fullerenes and the nanotubes. Still, it provides a rather good description of the confined $\mathrm{C}_{60}$ chains from a dynamical point of view, giving quantitative information in very good agreement with those known for solid $\mathrm{C}_{60}$. It appears reasonable to estimate that in the temperature range considered in this study, the presence of the surrounding nanotube has no effect other than providing a smooth one-dimensional container for the $\mathrm{C}_{60}$ chains. In other words, one can consider that any term in Eq. (1) which would eventually couple the longitudinal displacements of the confined fullerenes with the nanotube is negligible in the temperature range considered in this study. We believe that this stands only for some peculiar regions of the (pressure, temperature, tube diameter) phase space of the peapod system. In particular, we anticipate that the coupling between the nanotube and the $\mathrm{C}_{60}$ will become important at low temperature or high pressure, and that the longitudinal dynamics will be affected as theoretically predicted and observed for the rotational state of the confined chain. $2,3,19,20$

\section{v. CONCLUSION}

We have followed the evolution of the longitudinal dynamics of the $\mathrm{C}_{60}$ chains confined inside carbon nanotubes, by taking advantage of the partial orientation of the chains parallel to the plane of the sample in the form of a pellet. We find that the behavior of the $\mathrm{C}_{60}$ chains for $260<T<600 \mathrm{~K}$ is that of a $1 \mathrm{D}$ harmonic crystal, composed of a number
$N \sim 2700$ molecules (total length $\sim 2.7 \mu \mathrm{m}$ ), and with sound velocity $v=3.5 \mathrm{~km} \mathrm{~s}^{-1}$. From these physical characteristics, we understand the solidlike behavior of the chains at ambient temperature as resulting from the limited number of molecules per chain. The latter is too small to give the chains a liquidlike character as observed in harmonic atomic chains like $\mathrm{Hg}_{3-\delta} \mathrm{AsF}_{6}$, for example. When heated, the crystalline chain progressively melts into a one-dimensional liquid, which is characterized by the absence of elastic scattering at $Q_{1}=$ $2 \pi / d$ and a softer longitudinal dynamics with a sound velocity reduced by half the value in the crystalline phase. The melting process is progressive and takes place in a $\sim 150$-K temperature range around $600 \mathrm{~K}$. These observations are consistent with the strong change in the thermal expansion of the chain observed previously $^{21}$ using $\mathrm{x}$-ray diffraction in the same temperature range. The melting is caused by a combination of the linear increase of thermal fluctuations together with the increasing anharmonicity of the system.

\section{ACKNOWLEDGMENTS}

The authors acknowledge H. Mutka and M. Jimeñez-Ruiz (ILL) for stimulating discussions on the physics of reduced dimensionality and the diffusion in liquids. Financial support from EU through the NMI3 program is acknowledged. C.B. thanks the ILL and the LPS for financial support. The authors are indebted to the ILL and the FRM2 neutron facilities for the beam time allocated. V.D. thanks the Russian Foundation for Basic Research (Grant No. 12-03-00787) for financial support.

\section{APPENDIX: DATA TREATMENT}

If obtaining the sole $\mathrm{C}_{60}$ chains signal can a priori be obtained by a simple subtraction of the para and ortho data, other correcting factors have to be rigorously taken care of. Indeed, the sample absorption is not the same in both configurations, which affects both elastic and inelastic scattering. Moreover, the elastic intensity differs from one configuration to the other due to the anisotropy of the sample. ${ }^{29}$ A simple subtraction of the para and ortho data is therefore not correct, and we have to account for these intensity variations.

Complete data treatment is done as follows: (i) Raw data are normalized to the monitor counts, to vanadium (detector efficiency), subject to the various spectrometer dependent corrections, and transformed to $S(Q, \omega)$. (ii) For each configuration, ortho $(\perp)$ and para $(\|)$, and each $Q$ of the region of interest, the spectra are fitted using Lorentzian functions (inelastic contribution $I_{\text {inel }}$ ) and a Dirac peak convoluted with experimental resolution (elastic contribution $I_{\mathrm{el}}$ ). (iii) Using these fitted intensities, one performs the following operation in order to obtain the sole $\mathrm{C}_{60}$ signal:

$$
\begin{aligned}
I_{\mathrm{C}_{60}}(Q, \omega)= & I_{\text {inel }}^{\|}(Q, \omega)-\alpha \times I_{\text {inel }}^{\perp}(Q, \omega) \\
& +I_{\mathrm{el}}^{\|}(Q, \omega)[1-c(Q)],
\end{aligned}
$$

where $\alpha$ is the coefficient that corrects for the difference of absorption in both configurations, and $c(Q)$ the one that corrects for the different elastic scattering due to the sample's anisotropy. In the $Q$ areas of the region of interest where the $\mathrm{C}_{60}$ contribution is negligible, i.e., $Q<0.6 \AA^{-1}$ and 
$Q>0.76 \AA^{-1}$, we consider that the tubes' contribution does not evolve from one configuration to the other, and $c(Q)=1$. For $Q \in[0.6,0.76] \AA^{-1}$, we have to account for the elastic intensity in para configuration that is added to the one of the ortho configuration (giving the elastic intensity coming from the tubes), and therefore $c(Q)=\alpha I_{\mathrm{el}}^{\perp}(Q) / I_{\mathrm{el}}^{\|}(Q)$.

After this treatment, we obtain the sole $\mathrm{C}_{60}$ chains signal, containing both elastic and inelastic contributions.
*Corresponding author: rols@ill.fr

†Corresponding author: pascale.launois@u-psud.fr

${ }^{1}$ B. Smith, M. Monthioux, and D. Luzzi, Nature (London) 396, 323 (1998).

${ }^{2}$ K. H. Michel, B. Verberck, and A. V. Nikolaev, Phys. Rev. Lett. 95, 185506 (2005).

${ }^{3}$ K. H. Michel, B. Verberck, and A. V. Nikolaev, Eur. Phys. J. B 48, 113 (2005).

${ }^{4}$ C. Carraro, Phys. Rev. B 61, 16351 (2000).

${ }^{5}$ M. Hodak and L. A. Girifalco, Phys. Rev. B 64, 035407 (2001).

${ }^{6}$ M. M. Calbi, S. M. Gatica, and M. W. Cole, Phys. Rev. B 67, 205417 (2003).

${ }^{7}$ L. Girifalco and M. Hodak, Appl. Phys. A: Mater. Sci. Proc. 76, 487 (2003).

${ }^{8}$ B. Verberck, J. Cambedouzou, G. Vliegenthart, G. Gompper, and P. Launois, Carbon 49, 2007 (2011).

${ }^{9}$ H. Kataura, Y. Maniwa, M. Abe, A. Fujiwara, T. Kodama, K. Kikuchi, H. Imahori, Y. Misaki, S. Suzuki, and Y. Achiba, Appl. Phys. A: Mater. Sci. Proc. 74, 349 (2002).

${ }^{10}$ M. Chorro, S. Rols, J. Cambedouzou, L. Alvarez, R. Almairac, J.-L. Sauvajol, J.-L. Hodeau, L. Marques, M. Mezouar, and H. Kataura, Phys. Rev. B 74, 205425 (2006).

${ }^{11}$ S. Kawasaki, T. Hara, T. Yokomae, F. Okino, H. Touhara, H. Kataura, T. Watanuki, and Y. Ohishi, Chem. Phys. Lett. 418, 260 (2006).

${ }^{12}$ M. Chorro, J. Cambedouzou, A. Iwasiewicz-Wabnig, L. Noé, S. Rols, M. Monthioux, B. Sundqvist, and P. Launois, Eur. Phys. Lett. 79, 56003 (2007).

${ }^{13}$ C. Caillier, D. Machon, A. San-Miguel, R. Arenal, G. Montagnac, H. Cardon, M. Kalbac, M. Zukalova, and L. Kavan, Phys. Rev. B 77, 125418 (2008).

${ }^{14}$ S. Rols, R. Papoular, V. Davydov, A. Rakhmanina, C. Autret, and V. Agafonov, Fullerenes, Nanotubes, Carbon Nanostruct. 18, 412 (2010).

${ }^{15}$ T. Pichler, A. Kukovecz, H. Kuzmany, H. Kataura, and Y. Achiba, Phys. Rev. B 67, 125416 (2003).

${ }^{16}$ D. Neumann, J. Copley, R. Cappelletti, W. Kamitakahara, R. Lindstrom, K. Creegan, D. Cox, W. Romanow, N. Coustel, J. McCauley, Jr. et al., Phys. Rev. Lett. 67, 3808 (1991).

${ }^{17}$ P. Heiney, J. Phys. Chem. Solids 53, 1333 (1992).

${ }^{18}$ W. David, R. Ibberson, T. Dennis, J. Hare, and K. Prassides, Europhys. Lett. 18, 219 (1992).

${ }^{19}$ S. Rols, J. Cambedouzou, M. Chorro, H. Schober, V. Agafonov, P. Launois, V. Davydov, A. V. Rakhmanina, H. Kataura, and J.-L. Sauvajol, Phys. Rev. Lett. 101, 065507 (2008).

${ }^{20}$ E. Abou-Hamad, Y. Kim, T. Wågberg, D. Boesch, S. Aloni, A. Zettl, A. Rubio, D. E. Luzzi, and C. Goze-Bac, ACS Nano 3, 3878 (2009).

${ }^{21}$ C. Bousige, S. Rols, E. Paineau, S. Rouzière, C. Mocuta, B. Verberck, J. P. Wright, H. Kataura, and P. Launois, Phys. Rev. B 86, 045446 (2012).
${ }^{22}$ K. Matsuda, Y. Maniwa, and H. Kataura, Phys. Rev. B 77, 075421 (2008).

${ }^{23}$ Meijo, www.meijo-nano.com/en/products/swnt/index.html, Nagoya, Japan.

${ }^{24}$ MER, www.mercorp.com, Tucson, AZ.

${ }^{25}$ H. Kataura, Y. Maniwa, T. Kodama, K. Kikuchi, K. Hirahara, and K. Suenaga, Synth. Met. 121, 1195 (2001).

${ }^{26}$ L. G. Khvostantsev, L. F. Vereshchagin, and A. P. Novikov, High Temp.-High Press. 9, 637 (1977).

${ }^{27}$ J. Cambedouzou, V. Pichot, S. Rols, P. Launois, P. Petit, R. Klement, H. Kataura, and R. Almairac, Eur. Phys. J. B 42, 31 (2004).

${ }^{28}$ C. Bousige, Ph.D. thesis, Université Paris XI Orsay, 2012.

${ }^{29}$ R. Almairac, J. Cambedouzou, S. Rols, and J.-L. Sauvajol, Eur. Phys. J. B 49, 147 (2006).

${ }^{30}$ G. Squires, Thermal Neutron Scattering (Cambridge University Press, Cambridge, England, 1978).

${ }^{31}$ C. Bousige, S. Rols, J. Cambedouzou, B. Verberck, S. Pekker, E. Kováts, G. Durkó, I. Jalsovsky, E. Pellegrini, and P. Launois, Phys. Rev. B 82, 195413 (2010).

${ }^{32}$ The scattered intensity is the sum of the coherent and incoherent $S(Q, \omega)$ weighted by the scattering lengths, with $\sigma_{C}^{\text {coh }}=5.551$ barns, $\sigma_{C}^{\text {inc }}=0.001$ barns. Note also that $\sigma_{H}^{\text {coh }}=1.756$ barns and $\sigma_{H}^{\text {inc }}=80.26$ barns: minimization of the amount of hydrogenated pollutant was necessary for our analysis of the coherent scattering from the $\mathrm{C}_{60}$ chains, as underlined in Sec. II A.

${ }^{33}$ S. Rols, H. Jobic, and H. Schober, C. R. Phys. 8, 777 (2007).

${ }^{34}$ I. U. Heilmann, J. D. Axe, J. M. Hastings, G. Shirane, A. J. Heeger, and A. G. MacDiarmid, Phys. Rev. B 20, 751 (1979).

${ }^{35}$ N. Ashcroft and N. Mermin, Solid State Physics (Holt-Saunders, New York, 1976).

${ }^{36}$ T. Yoshida, K. Shobu, and H. Mori, Progr. Theor. Phys. 66, 759 (1981).

${ }^{37}$ S. Lovesey, Theory of Neutron Scattering from Condensed Matter (Clarendon, Oxford, 1984).

${ }^{38}$ R. Jones, Acta Crystallogr. 2, 252 (1949).

${ }^{39}$ V. J. Emery and J. D. Axe, Phys. Rev. Lett. 40, 1507 (1978).

${ }^{40}$ H. J. Mikeska, Solid State Commun. 13, 73 (1973).

${ }^{41}$ P. A. Albouy, J. P. Pouget, and H. Strzelecka, Phys. Rev. B 35, 173 (1987).

${ }^{42}$ F. Lindemann, Z. Phys. 11, 609 (1910).

${ }^{43} \mathrm{~A}$ phase transition in a $1 \mathrm{D}$ system can occur in the case of long-range interactions (Ref. 44).

${ }^{44}$ E. Lieb and D. Mattis, Mathematical Physics in One Dimension: Exactly Soluble Models of Interacting Particles (Academic, New York, 1966).

${ }^{45}$ The Heisenberg incertitude results in the relation $t \mathrm{ps} \simeq$ $0.658 / \hbar \omega \mathrm{meV}$. Therefore, a relaxation time of $50 \mathrm{ps}$ corresponds to a full width at half maximum of $26 \mu \mathrm{eV}$.

${ }^{46}$ Y. Soifer, N. Kobelev, and V. Levin, J. Alloys Compd. 310, 292 (2000). 